\title{
Auricular Cartilage Interposition Arthroplasty in Recurrent Temporomandibular Joint Ankylosis in Pediatric Patients
}

\author{
Ahmad Liaquat ${ }^{1}$, Arun Kumar Shah ${ }^{2}$, Nabeela Riaz ${ }^{3}$ \\ ${ }^{1}$ Assistant Professor, Department of Oral and Maxillofacial Surgery, University College of Medicine and Dentistry, \\ University of Lahore, Pakistan \\ ${ }^{2}$ Senior Lecturer, Department of Oral and Maxillofacial Surgery, Chitwan Medical College, Nepal \\ ${ }^{3}$ Professor \& Head, Department of Oral and Maxillofacial Surgery, King Edward Medical University, Lahore, Pakistan
}

\begin{abstract}
AB S TR ACT
Background: Temporomandibular joint (TMJ) ankylosis in early childhood can lead to disturbances in growth, facial asymmetry and difficulties in eating as well as in breathing. The objective of the study was to evaluate the effectiveness of auricular cartilage as an interpositional material after gap arthroplasty in pediatric patients with temporomandibular joint ankylosis in maintaining the achieved postoperative mouth opening.

Material and Methods: This prospective study was conducted at the Oral and Maxillofacial Surgery Department of King Edward Medical University, Lahore, from 1st January 2019 to 30th November 2019. Thirty patients, aged 5 to 15 years with clinical and radiological diagnosis of TMJ ankylosis were included in the study. TMJ ankylotic mass was removed, the gap was created and interposition of auricular cartilage graft was done in these patients. Mouth opening was measured as a distance between the incisal edges of mandibular central incisors to maxillary central incisors using the scale. Preoperative and postoperative mouth opening was noted and compared using the paired t-test.

Results: Among the thirty patients, 19 were male and 11 were female. The mean $( \pm S D)$ age of patients was $9.23 \pm 3.23$ years (age range 5 to 15 years). The mean preoperative mouth opening (T1) was $7.3 \pm 3.1 \mathrm{~mm}$, while the mean postoperative mouth opening (T2) was $29.2 \pm 3.3 \mathrm{~mm}$. The mean interincisal distance was $29.0 \mathrm{~mm}$ (range $21 \mathrm{~mm}$ to 35 $\mathrm{mm}$ ). The mean difference in pre- and postoperative mouth opening (T2-T1) was $21.6 \pm 5 \mathrm{~mm}$.

Conclusions: Autogenous auricular cartilage graft is an efficientand reliable interpositional material for achieving early postoperative mouth opening in TMJ ankylosis patients.

Key words: Auricular cartilage graft, Temporomandibular joint ankylosis, Trismus.

Authors' Contribution: Correspondence:

${ }^{1}$ Conception; Literature research; manuscript Ahmad Liaquat

design and drafting; 2,3 Critical analysis and Email:ahmadliaquat@hotmail.com

manuscript review; Data analysis; Manuscript

Editing.

Article info:

Received: May 2, 2020

Accepted: March 3, 2021
\end{abstract}

Cite this article. Liaquat A, Shah AK, Riaz N. Auricular Cartilage Interposition Arthroplasty in Recurrent Temporomandibular Joint Ankylosis in Pediatric Patients. J Islamabad Med Dental Coll. 2021; 10(1): 9-14. Doi: 10.35787/jimdc.v10i1.545

\section{Introduction}

Temporomandibular joint ankylosis (TMJ) is characterized by difficulty or limited mouth opening. TMJ ankylosis is associated with feeding problems, occlusal disharmony, upper airway obstruction, inability to maintain oral hygiene, dental caries, and facial asymmetry in developing children. ${ }^{1}$ In TMJ 
ankylosis, the condyle of mandible fuses with the articular surface of the temporal bone and limits the mouth opening. Development of TMJ ankylosis in childhood causes facial disfigurement, nutritional imbalances due to inability to eat food and psychological problems. ${ }^{1}$ Trauma to the mandible or TMJ is the leading cause of ankylosis in paedriatic population (13-100\%). ${ }^{2}$ Other etiological factors include systemic or local infection (40\%) or systemic diseases (10\%), such as rheumatoid arthritis, ankylosing spondylitis, and psoriasis in the adult patient. ${ }^{2}$ Orhan Guven described a higher rate of post-traumatic ankylosis in children. ${ }^{3}$ A study conducted in Pakistan showed peak incidence of TMJ ankylosis in 6-10 years and the most common causes for TMJ ankylosis were reported as fall (82.8\%) and dysphonia (66.7\%) due to impairment of upper airways. Dysphasia, facial deformity, and psychological disturbances are consequences of TMJ ankylosis. $^{2,4}$

Treatment of TMJ ankylosis is very challenging because of frequent recurrences. ${ }^{5}$ However, gap arthroplasty and insertion of interposition material to prevent re-ankylosis is the mainstay of treatment. Gap arthroplasty without interposition results in recurrence of ankylosis in almost $53 \%$ of patients. ${ }^{2}$ Timing of the surgery and choice of the interposition material may vary from center to center, but the main treatment is the same. Interpositional materials separate the condylar area from the glenoid fossa, so their anchorage and viability are very important. ${ }^{6}$

Various types of interposition materials have been used in the literature for augmentation of height of ramus and prevention of re-ankylosis. These materials are autologous grafts (rib grafts, auricular cartilage, temporalis fascia etc.) and alloplastic material (silastic material, custom-made titanium condylar prosthesis etc.). Temporalis fascia with or without temporalis muscle is the most commonly used interpositional material because of its proximity to the operating site and being a pedicled flap, it can resist the wear and tear forces. ${ }^{7,8}$

Auricular cartilage is another reliable graft present very close to the operating site and has been used as an interposition graft after TMJ ankylosis surgery with good results. ${ }^{8}$ The main advantages include safety, viability, preserving the function of the condyle, non-antigenicity and low donor site morbidity. Availability of auricular graft adjacent to the surgical field makes it the most attractive choice for surgeons. ${ }^{9}$ Auricular cartilage is fixed to the condyle by sutures.

The rationale of this study was to evaluate the auricular cartilage as an interpositional material in terms of maintaining the postoperative mouth opening. Although different types of autogenous graft and alloplastic material are used for interposition, no material has produced uniformly successful results. Limited data is available on the use of auricular cartilage as an interpositional material in recurrent TMJ ankylosis cases. Moreover, a previous study using auricular cartilage was conducted on a small number of patients $(n=10) .{ }^{9}$ So the present study was designed to generate more reliable results for our local population.

\section{Material and Methods}

This prospective study was conducted at the Oral and Maxillofacial Surgery Department of King Edward Medical University, Lahore, Pakistan from $1^{\text {st }}$ January 2019 to $30^{\text {th }}$ November 2019. Approval for the study was obtained from the ethical review board of the university (Reference number 1697/18). Patients aged 5 to 15 years with unilateral and bilateral or recurrent TMJ ankylosis were included in the study. Patients with an ear deformity or medically unfit for surgery were excluded.

After explaining the outcome of surgical procedures to every patient, informed consent was taken from 
their parents/guardians before the scheduled surgery. To diagnose TMJ ankylosis, orthopantomograms and computer tomography was done. For removal of fibrous adhesions or ankylotic mass, preauricular incision was used. Dissection was done layer by layer to reach up to the ankylotic mass, preserving the zygomatic and temporal branches of the facial nerve. Ankylotic mass was removed and auricular cartilage graft was harvested from the posterior aspect of the helix. The auricular cartilage was secured with condyle using proline sutures. Postoperative management was done in the ward until the removal of sutures. Mouth opening was measured from the incisal edges of mandibular central incisor to maxillary central incisor by the scale. Mouth opening was measured preoperatively (T1) and recorded. Postoperative mouth opening was measured after three months (T2). The mean difference of mouth opening (T2-T1) was calculated for each patient, using the paired t-test with $P$-value $<.05$ considered as statistically significant.

\section{Results}

Among the thirty patients included in this study 19 were males and 11 were females. The mean ( \pm SD) age of the patients was $9.23 \pm 3.23$ years. The mean preoperative mouth opening (T1) was $7.3 \pm 3.1 \mathrm{~mm}$. There was no significant difference among male and female patients in terms of preoperative mouth opening ( $P=.29$ ). The mean postoperative mouth opening (T2) was $29.2 \pm 3.3 \mathrm{~mm}$, with no significant difference among male and female patients $(P=$ .99). The mean difference in pre- and postoperative mouth opening (T2-T1) was $21.6 \pm 5 \mathrm{~mm}$, which was statistically significant $(P<.001)$. Seventeen patients (56.66\%) had unilateral TMJ ankylosis with $58.82 \%$ ( $n=10)$ having right-sided and $41.17 \%(n=7)$ patients having left-sided TMJ ankylosis. Thirteen patients (43.33\%) had bilateral TMJ ankylosis. The most common cause of TMJ ankylosis was history of fall
(80\%), followed by roadside accidents (13.33\%) and birth trauma (6.67\%).

\begin{tabular}{|l|c|c|}
\hline \multicolumn{3}{|c|}{$\begin{array}{c}\text { Table I: Gender wise comparison of pre- and } \\
\text { postoperative mouth opening }\end{array}$} \\
\hline & Male & Female \\
\hline Mean Age (years) & $9.47 \pm 3.2$ & $8.82 \pm 3.4$ \\
\hline $\begin{array}{l}\text { Mean preoperative } \\
\text { mouth opening } \\
\text { (mm) }\end{array}$ & $7.6 \pm 3.5$ & $7.8 \pm 2.4$ \\
\hline $\begin{array}{l}\text { Mean post- } \\
\text { Operative mouth } \\
\text { opening (mm) }\end{array}$ & $29.8 \pm 3.3$ & $28.2 \pm 3$ \\
\hline $\begin{array}{l}\text { Mean difference in } \\
\text { mouth opening }\end{array}$ & $22.3 \pm 5.7$ & $20.4 \pm 3.2$ \\
\hline Paired t value & -22.3 & -20.4 \\
\hline $95 \% \mathrm{Cl}$ & -25.0 to -19.5 & -22.5 to -18.2 \\
\hline$P$-value & $<.001$ & $<.001$ \\
\hline
\end{tabular}

\begin{tabular}{|c|c|}
\hline \multicolumn{2}{|c|}{ Table II: Duration of TMJ ankylosis } \\
\hline Duration of ankylosis (years) & $\mathbf{n}(\%)$ \\
\hline $1-2$ & $8(26.67)$ \\
\hline $2-4$ & $13(43.33)$ \\
\hline$>5$ & $9(30)$ \\
\hline Total & 30 \\
\hline
\end{tabular}

\section{Discussion}

This study aimed to evaluate auricular cartilage as an interpositional material in gaining the postoperative mouth opening. TMJ is a main part of the joint of the temporal region. Ankylosis of the joint in a growing child may result in disfigurement of the face, difficulty in eating, speech, and maintaining oral hygiene. TMJ ankylosis can result in retrognathic mandible, causing cosmetic and functional deformities. The main objective of treatment is to correct the jaw opening and prevent the development of disfigurement and psychological distress. ${ }^{10}$

TMJ ankylosis is classified according to the type of tissue involved (bony, fibrous, or both), location 
(intracapsular or extracapsular), and extent of fusion (incomplete or complete). ${ }^{11}$ In children, the head of the condyle is small, so its injury can result in hemarthrosis. Pain causes the hypomobility of the joint, which in turn favors bone formation leading to ankylosis. ${ }^{12} \mathrm{TMJ}$ can be infected by infection of the ear or distal spread from scarlet fever or tuberculosis. ${ }^{13}$

Trauma to the mandible and in the TMJ region is the leading cause of ankylosis in children. Many cases with previous history of trauma to the chin or a history of fall and trauma to jaws in childhood develop trismus later on in life. ${ }^{14}$ They become nutritionally deficient and trismus also poses a great hindrance in maintaining good oral hygiene. So, these patients have severe malaligned teeth and caries. Due to mandibular retrognathism and facial disfigurement, these patients are socially rejected and develop psychological issues.

Many graft materials are available, which can be classified as autologous or alloplastic. Grafts from the clavicle, iliac crest, costochondral, auricular cartilage and dermal fat are considered autologous grafts. ${ }^{15}$ Alloplastic grafts include silicon, protoplast, and acrylic, etc. ${ }^{15}$ Alloplastic material have adverse effects like foreign body reactions, infection, instability, and extrusion. Therefore, alloplastic material is not the preferred choice. Among the autogenous materials, cartilage has many physiological and mechanical advantages. It is available within the same operative field with less donor site morbidity. The study by Krishnan showed that the shape of the cartilage is well-fitting the condylar process, and maintaining its viability thus helping in the condyle function. He reported temporary facial nerve paralysis in two patients. ${ }^{9}$ In our study, temporary facial nerve paralysis was observed in five patients. Postoperative use of steroids resolved the facial injury and only one patient had permanent damage to the frontal branch of the facial nerve. The injury occurred during surgery as branches of the facial nerve were in the course of the surgical site. It was not related to the choice of graft. There was no donor-site morbidity noted in our cases. In this study, 30 patients were included, out of which $63 \%$ were male and $37 \%$ were female. Ten patients had recurrent TMJ ankylosis. The patients' age ranged from 5 to 15 years with a mean $\pm S D$ of $9.23 \pm 3.23$ years, which is comparable to the study conducted by Gupta et al., where the mean \pm SD age was $11.1 \pm 3.3$ years. ${ }^{16}$ Similarly, out of 87 patients reported from the Orthodontic and Oral and Maxillofacial Surgery departments of Khyber College of Dentistry, 63.2\% were male and $35.6 \%$ were female, ranging from 2 to 16 years. ${ }^{1}$ However, Svensson et al. reported 18 patients with 15 females and three males and a mean age of 39 years. ${ }^{17}$

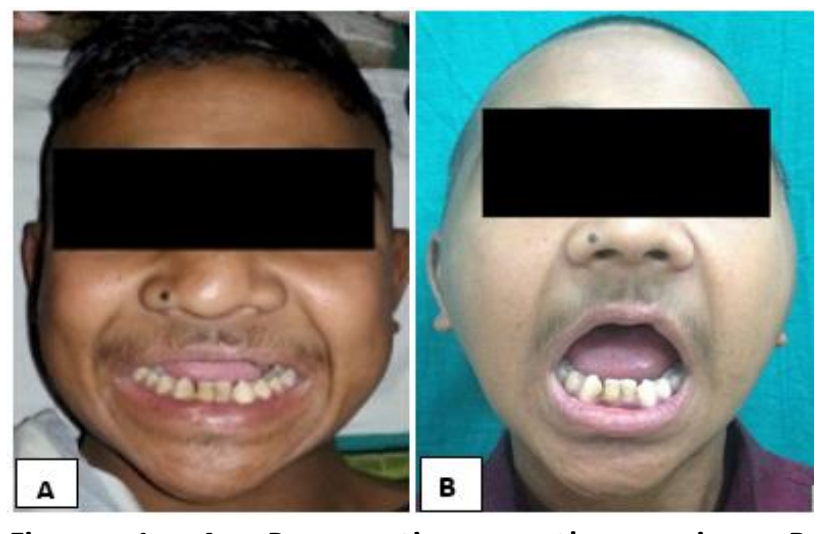

Figure 1. A: Preoperative mouth opening. B: Postoperative mouth opening in the same patient.

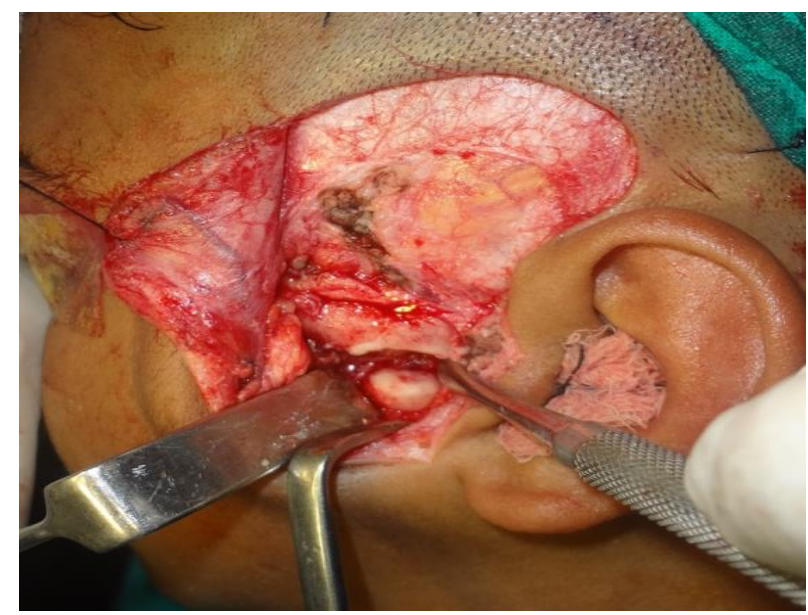

Figure 2: Exposure of ankylotic mass by preauricular incision. 


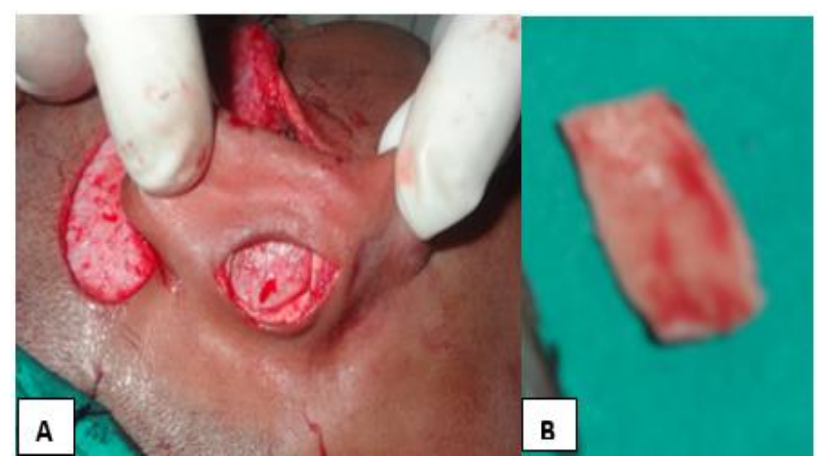

Figure 3: A shows harvesting of auricular cartilage, while $B$ reveals harvested cartilage.

Krishnan reported the mean preoperative mouth opening to be $2.5 \mathrm{~mm}$ in his study while Svensson et al. reported $28 \mathrm{~mm} \cdot{ }^{9,17}$ Muller et al. reported the preoperative mouth opening to be $17 \mathrm{~mm}$ in their single patient study. ${ }^{18}$ In the present study, the preoperative mean mouth opening was $7.6 \pm 3.5 \mathrm{~mm}$ for males and $7.8 \pm 2.4 \mathrm{~mm}$ for females and the range of mouth opening preoperatively for males and females was 3-15 $\mathrm{mm}$ and 4-12 $\mathrm{mm}$ respectively with a mean preoperative mouth opening of 7.7 $\mathrm{mm}$. This is somewhat comparable to the study by Krishnan ${ }^{9}$ but varies considerably from figures reported by Svensson et al. ${ }^{17}$

The mean postoperative mouth opening in our study was $29.2 \mathrm{~mm}$, which is comparable to $29.6 \mathrm{~mm}$ reported by Krishnan. ${ }^{9}$ Other studies report variable figures of $5 \mathrm{~mm}$ (Muller et al.) ${ }^{18}$ and $35 \mathrm{~mm}$ (Svensson et al.), ${ }^{17}$ respectively. The postoperative mouth opening of all these studies is comparable. ${ }^{9}$ The similarity in the postoperative mouth opening but the variability in preoperative mouth opening may be because of the time of surgery and duration of TMJ ankylosis. We followed-up our patients for two years, but after that many patients were lost to follow-up.

The most frequent etiology of TMJ ankylosis in the pediatric population is fall history. ${ }^{1}$ In our study, $80 \%$ of patients had ankylosis secondary to fall history.
Children do not perform postoperative physiotherapy regularly that resulted in re-ankylosis in our cases. These patients should be put on a longterm follow-up to see re-ankylosis. We found this graft very useful in recurrent cases of TMJ ankylosis where temporalis fascia or muscles have already been used, so we recommend its use in recurrent cases of TMJ ankylosis. Future studies are suggested to explore its long-term effects in maintaining the jaw opening.

\section{Conclusion}

An autogenous auricular cartilage graft is good interpositional material for the successful treatment of TMJ ankylosis pediatric patients in terms of achieving adequate postoperative mouth opening.

\section{References}

1. Murad N, Rasool G. Trauma as a Most Common Cause of TMJ Ankylosis. J Pak Oral Dent J. 2011; 31 (1): 45-47.

2. Khan ZA, Alam J, Khan S, Abid H, Warriach RA. Correlation between Childhood Chin Trauma, Condylar Fracture and TMJ Ankylosis. Pak Oral Dent J. $2010 ; 30$ (1): 47-51.

3. Güven O. A clinical study on temporomandibular joint ankylosis in children. J Craniofac Surg. 2008; 19(5): 1263-9. Doi: 10.1097/SCS.0b013e318157 $7 b 1 b$.

4. Shetty P, Thomas A, Sowmya B. Diagnosis of temporomandibular joint (TMJ) ankylosis in children. J Indian Soc Pedod Prev Dent. 2014; 32(3): 266. Doi: 10.4103/0970-4388.135848.

5. Chen S, He Y, An JG, Zhang Y. Recurrence-Related Factors of Temporomandibular Joint Ankylosis: A 10Year Experience. J Oral Maxillofac Surg. 2019; 77(12): 2512-21. Doi: 10.1016/j.joms.2019.06.172.

6. Goswami D, Singh S, Bhutia O, Baidya D, Sawhney C. Management of young patients with temporomandibular joint ankylosis-a surgical and anesthetic challenge. Indian J Surg. 2016; 78(6): 4829. Doi: 10.1007/s12262-016-1551-5.

7. Guruprasad Y, Chauhan DS, Cariappa KM. A retrospective study of temporalis muscle and fascia flap in treatment of TMJ ankylosis. J Maxillofac Oral Surg. 2010; 9(4): 363-8. Doi: 10.1007/s12663-0100139-z. 
8. Makwana R, Ranadive $P$, Deshpande $M$. Use of temporalis fascia flap in the treatment of Temporomandibular Joint ankylosis-a clinical audit of 5 years. Int J Oral Maxillofac Surg. 2015; 44(Supp 1): e108.Doi: 10.1016/j.ijom.2015.08.691.

9. Krishnan B. Autogenous auricular cartilage graft in temporomandibular joint ankylosis-an evaluation. Oral Maxillofac Surg. 2008; 12(4):189-93. Doi: 10.1007/s10006-008-0136-2.

10. Gulsen A, Sibar S, Ozmen S. Orthognathic treatment of facial asymmetry due to temporomandibular joint ankylosis. Arch Plast Surg. 2018; 45(1): 74-9. Doi: 10.5999/aps.2016.01774.

11. He D, Yang C, Chen M, Zhang X, Qiu Y, Yang X, et al. Traumatic temporomandibular joint ankylosis: our classification and treatment experience. J Oral Maxillofac Surg. 2011; 69(6): 1600-7. Doi: 10.1016/j.joms.2010.07.070.

12. Ramly EP, Jason WY, Eisemann BS, Yue O, Alfonso AR, Kantar RS, et al. Temporomandibular joint ankylosis in pediatric patients with craniofacial differences: causes, recurrence and clinical outcomes. J Craniofac Surg. 2020; 31(5): 1343-7. Doi: 10.1097/SCS .0000000000006328 .

13. He D, Ellis III E, Zhang Y. Etiology of temporomandibular joint ankylosis secondary to condylar fractures: the role of concomitant mandibular fractures. J Oral Maxillofac Surg. 2008; 66(1): 77-84. Doi: 10.1016/j.joms.2007.08.013.

14. Kaban LB, Bouchard C, Troulis MJ. A protocol for management of temporomandibular joint ankylosis in children. J Oral Maxillofac Surg. 2009; 67(9): 1966 78. Doi: 10.1016/j.joms.2009.03.071.

15. Park MW, Eo MY, Seo BY, Nguyen TT, Kim SM. Gap arthroplasty with active mouth opening exercises using an interocclusal splint in temporomandibular joint ankylosis patients. Maxillofac Plast Reconstr Surg. 2019; 41: 18. Doi: 10.1186/s40902-019-0200-x.

16. Gupta VK, Mehrotra D, Malhotra S, Kumar S, Agarwal GG, Pal US. An epidemiological study of temporomandibular joint ankylosis. Natl J Maxillofac Surg. 2012; 3(1): 25. Doi: 10.4103/09755950.102146.

17. Svensson B, Wennerblom K, Adell R. Auricular Cartilage Grafting in Arthroplasty of TMJ: a retrospective clinical follow-up. Oral Surg Oral Med Oral Pathol Oral Radiol Endod. 2010; 109(3): e1-e7. Doi: 10.1016/j.tripleo.2009.10.029.

18. Mueller CK, Thorwarth MW, Mosgau SS. Combined Treatment Protocol for TMJ Ankylosis. J Craniofac Surg. 2009; 20(1): 258-60. Doi: 10.1097/SCS.0b013e31818432fb. 